\title{
A description and evaluation of light-emitting diode displays for generation of visual stimuli*
}

\author{
PERRY M. NEALIS, ROBERT M. ENGELKE \\ and DOMINIC W. MASSARO $\dagger$ \\ University of Wisconsin, Madison. Wisconsin 53706
}

A description of the design and function of light-emitting diode (LED) display modules is given. A method is presented for the construction of LED display devices and their on-line use with a small real-time computer. An assessment was made of the perceptual confusion of the alphanumeric characters generated by the LED displays. Presenting the characters briefly so that recognition errors would occur, it was found that perceptual confusions between items were minimal for most characters. The few confusions that did occur could be predicted by the formal similarity of the characters.

The application of electronic components in experimental psychology laboratories has grown rapidly in the past 15 to 20 years. However, relatively few improvements have been made in the functional precision of peripherals in contrast to the dramatic innovations that have occurred in the design of control systems. The necessity of employing conventional peripheral devices has prevented full realization of the capabilities of small real-time computers and computer-based process control systems in the investigation of visual perceptual processes. Some optoelectronic devices currently in use exhibit response characteristics which are slow or inexact relative to computer speed and precision (see Haber, 1968).

Recent technological progress, however, has permitted more precise manipulation of visual stimuli through the use of display devices such as cathode ray tubes (CRTs) and gas discharge tubes. These instruments have better response characteristics than the conventional tachistoscope and rise-and-fall times (the duration of time required to increment from $10 \%$ to $90 \%$ luminance) which compare more favorably with the speeds at which computers execute instructions. With displays of this type, however, digital access is not always possible. These devices are also particularly sensitive to vibrations and are not always completely solid state. Unless alphanumeric CRTs with storage tubes are used, these optic devices require relatively complex logic systems for their implementation with on-line computer operations.

This investigation was supported in part by United States Public Health Service Grant MH-19399-01 to D. W. Massaro. The authors wish to thank Larry Winkler and John Hammerly for their assistance in carrying out the experiment.

tReprint requests should be addressed to Dominic $w$. Massaro, Department of Psychology, University of Wisconsin, Madison, Wisconsin 53706 .
Specifying alphanumeric readouts with the more common CRT display devices requires a sophisticated interface between current-voltage requirements of decoder-drivers and attainment of proper display brightness.

The most recent technological advances in optoelectronics results from U.S. efforts in the space program (Time, April 1972). Light-emitting diodes (LEDs) are examples of these spin-offs. LED display devices were originally used as instrument readouts in space vehicles and related equipment. This paper presents a description of their application in a computer-controlled auditory research laboratory.

\section{DESIGN AND OPERATION OF LED MODULES}

LEDs are made of semiconductors (e.g., diffused planar gallium arsenide phosphide) which glow as current passes through them. They require little power consumption, are sturdy, easily miniaturized (1/32 in. wide), and have almost instantaneous rise-and-fall characteristics. The Monsanto Company markets an alphanumeric display assembly, the MDA-III, ${ }^{1}$ which is a 36-diode, $5 \times 7$ dot matrix module with decimal point, having a character width and height of 6 and $8 \mathrm{~mm}$, respectively. Figure 1 presents a diagram of the functional components of the module. The MDA-III has self-contained character generator, decoder, clock generator, and buffer transistors which bring the system interface to a convenient location. Character selection is achieved by presenting a 6-bit binary word, conventional logic signals $(1 \geqslant+3.5 \mathrm{~V}$ and $0 \leqslant 0.6 \mathrm{~V})$, at the module input. Character generation takes place within a 2,240-bit read only memory (ROM) and is organized as $645 \times 7$ dot matrix characters with column-by-column outputs. The ROM is programmed with the American Standard Code for Information Interchange (ASCII), which allows character specification directly from the output buffer of a computer or computer-based control system. This 6-bit word is obtained by deleting the first digit of the three-digit octal code. For example, the letter $W$ is represented in ASCII as 327 , which then becomes 27 and can be specified as the 6-bit word, 010111 (cf. Fig. 1).

When the 6-bit binary inputs enter the ROM, the memory unit receiving a clock pulse responds by looking up the 7-bit word in its programmed memory. This 7-bit word is then presented to the ROM output. For each character, there are five 7-bit words stored in memory. Simultaneously and in time, the clock commands the scanner to enable the proper column of the array. A 


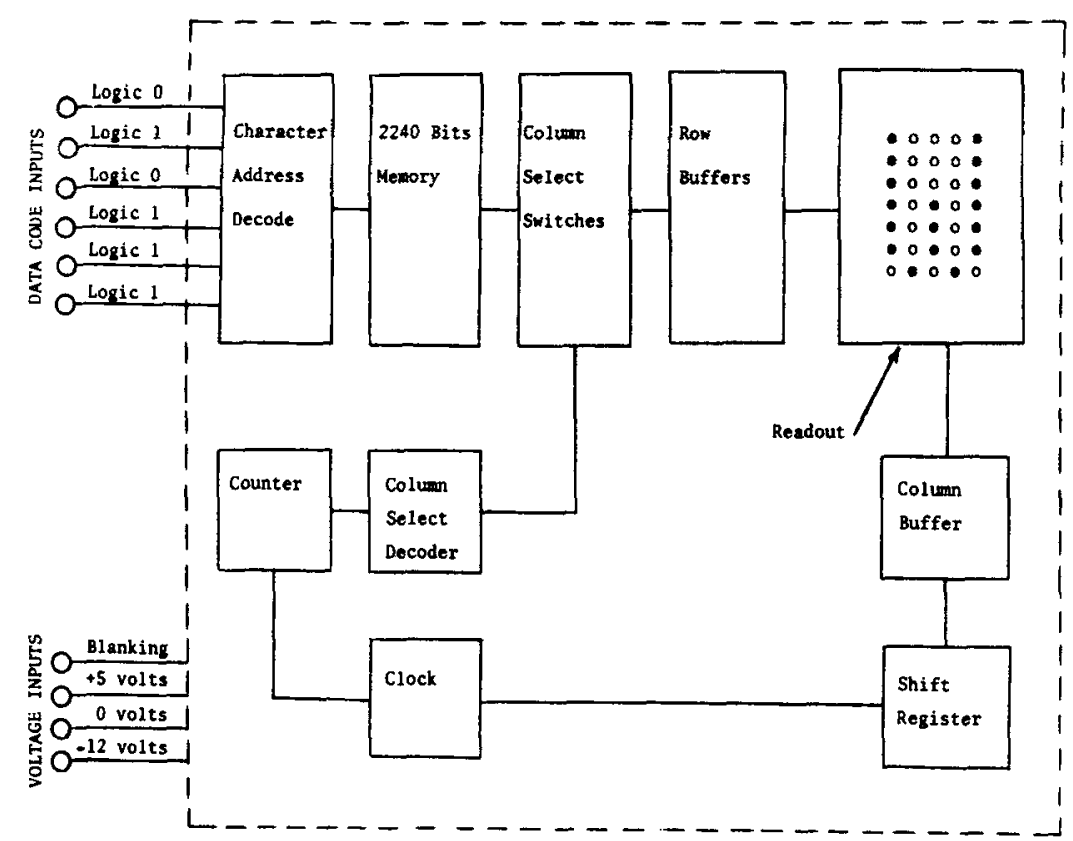

Fig. 1. Functional diagram of the MDA.III light-emitting diode display module. Character address for the letter $W$ is 27.

complete scan of the five-column matrix is executed within 750 microsec, with the rise-and-fall time for any particular LED or column of seven LEDs typically within 25 nsec. Additional tuning of the LED module permits a single character to be displayed within 10 microsec with a luminance of virtually $100 \%$. The character appears as a bright red image ( $300 \mathrm{fL}$ with peak emission wavelength of $650 \mathrm{~nm}$ ), which is minimally diffused by the clear epoxy lens of the MDA-III. In addition to these benefits, LED display modules eliminate the user's concern of readout specifications and permit the application of these displays with computer systems independent of the user's knowledge for interfacing purposes of electrooptical parameters.

\section{IMPLEMENTATION OF LED MODULES}

Our computer-controlled laboratory was designed to provide precise control of auditory stimuli through on-line operation of a PDP-8/L computer (Digital Equipment Corporation), which controls and monitors all experimental events (Massaro, 1972). Peripheral hardware equipment includes an analog-to-digital converter, a digital-to-analog converter, and a digitally controlled Wavetek Model 155 oscillator. The S's responses are made on a response panel connected to the computer input buffer. Responses are stored in memory core, and a printout of the appropriate confusion matrix can be obtained after test. Addition of the LED displays was accomplished by construction of four $S$ display consoles, each consisting of three MDA-III readouts arranged in side-by-side fashion and an interface system in which the pulse is latched and the logic stored indefinitely for the display modules. The four $\mathrm{S}$ display consoles are connected in parallel for simultaneous operation. The interface between the display modules and the computer consists of the three stages shown in Fig. 2: (1) a storage register which is connected to an

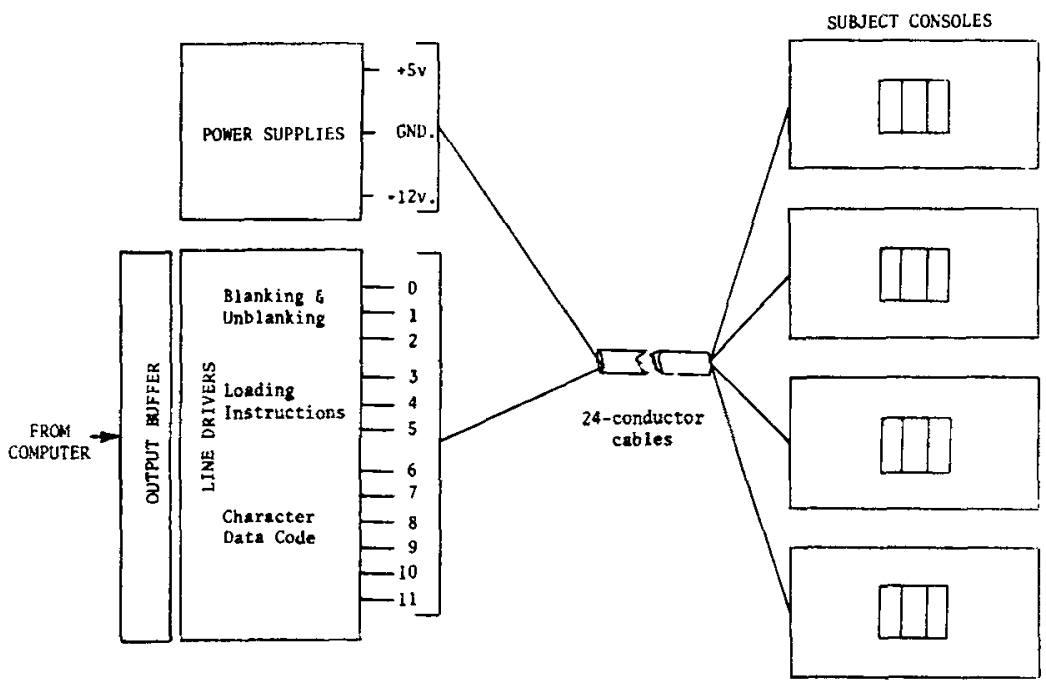

Fig. 2. Flowchart of LED display consoles on-line with PDP-8/L computer. Each console contains third-stage interface logic and three LED display modules. 
Fig. 3. Schematic representation of alphanumeric characters generated by MDA-III display modules. Each dot represents a single LED.

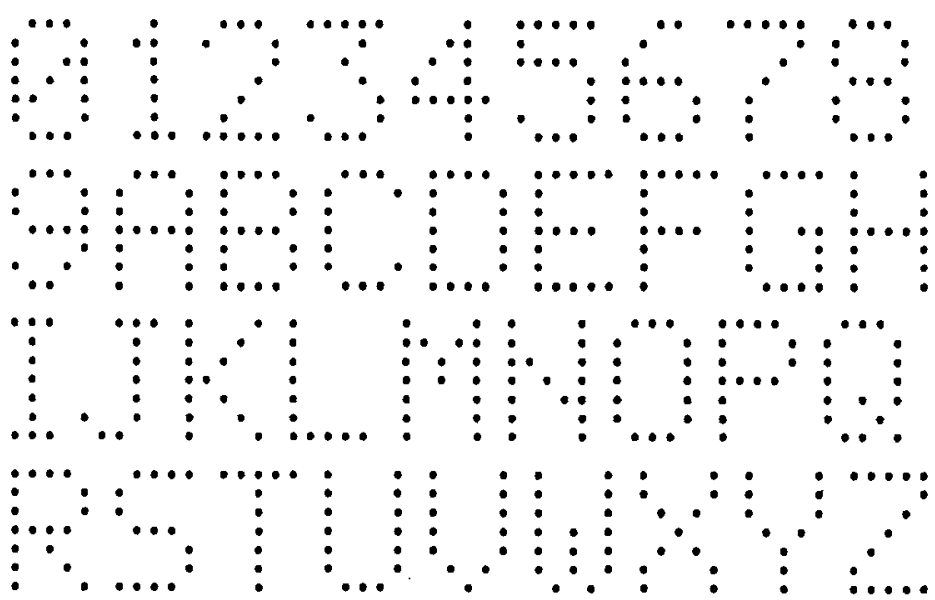

external bus, (2) a set of line drivers which transmits the storage register bits over long cables to the display consoles, and (3) the necessary signal conditioning and code storage circuitry for each display console.

The storage register and associated circuitry are capable of operating at the necessary speed for direct computer control. When the proper command is executed by the computer, the contents of the accumulator are placed in the output buffer, where the slower second and third interface stages have access to the information for a sufficient length of time. The second interface stage, consisting of 12 line drivers, transmits the contents of the output buffer over four multiconductor cables to the display consoles. Power for driving the displays and the third-stage interface logic is also fed through these cables $(+5,-12$, and $0 \mathrm{~V})$.

The logic system contained in each display console is the third interface stage, which shapes the incoming data and control signals for loading the 6-bit display storage registers. Since only 12 lines are available from the computer, all three 6-bit registers of each console cannot be loaded simultaneously. The 12 bits are assigned as follows: Bits 0, 1, and 2 contain blank (Logic 1) and unblank (Logic 0 ) signals for the left, middle, and right modules, respectively; Bits 3,4 , and 5 handle loading instructions (e.g., if Bit $3=$ Logic 1 and Bits 4 and $5=$ Logic 0 , then the leftmost LED module of each console is loaded); Bits 6-11 transmit the character specification code. All 12 lines are terminated at the display consoles with pull-up resistors to $5 \mathrm{~V}$. Additionally, a small capacitor was placed on Lines 3,4 , and 5 to ensure good electrical noise immunity and to cause a brief time delay of 50 microsec in the onset of the load instruction, which allows sufficient time for the data lines to settle.

\section{RECOGNITION OF LED-GENERATED CHARACTERS}

An experiment was conducted to determine whether or not the alphanumeric characters generated by the LED displays were reasonably discriminable and to assess the effects of perceptual confusion between stimuli. Figure 3 presents a schematic representation of the test stimuli generated by the LED modules. Each dot represents a single $\mathrm{LED}$.

\section{Subjects}

\section{Method}

The six males and six females employed in this study were recruited from the available pool of students enrolled in introductory psychology courses at the University of Wisconsin.

\section{Procedure}

The Ss were required to identify the letters $A$ through $Z$ and the numbers 0 through 9 , which were presented at random on the middle LED module. The test stimulus was displayed for $20 \mathrm{msec}$ and immediately followed by a series of masking stimuli presented in sequential order. The masking stimuli for the first six Ss consisted of the characters (\#), (@), $\left({ }^{*}\right)$, and $(\%)$. The masking stimuli for the second six Ss were (\%), (@), (\&), and (\#). In both conditions, the sequence of masking stimuli was presented twice on each trial. Since each mask was displayed for $10 \mathrm{msec}$, the total masking stimulus time was $80 \mathrm{msec}$. A trial consisted of the presentation of a single test character followed immediately by the masking characters. Each $\mathrm{S}$ was acquainted with the character configurations before test. The S's identification responses were made on a Teletype Model AS33 keyboard. The computer program allowed the Ss to self-pace the trials and each response, and provided an intertrial interval of $1 \mathrm{sec}$. Each $\mathrm{S}$ was tested individually on three blocks of 300 trials each, with 5-min rest intervals between trial blocks. The approximate viewing distance was $55 \mathrm{~cm}$, giving horizontal and vertical visual angles of 4 and $5.3 \mathrm{~min}$, respectively.

\section{Results and Discussion}

A confusion matrix of the recognition responses pooled over Ss and all 900 trials was obtained. The perceptual confusions between items were minimal for most stimuli, and a condensed list of maximally confused characters is presented in Fig. 4. The confusions can, for the most part, be predicted by the formal similarity of the letters shown in Fig. 3. It appears that MDA-III modules can be reliably employed in psychophysical tasks which require alphanumeric stimuli, providing some discretion is exercised by the $\mathrm{E}$ in selecting test characters.

Since all 36 diodes of the MDA-III module cannot be simultaneously illuminated, selection of masking stimuli is equally important. Investigators would find it 


\begin{tabular}{|c|c|c|c|c|c|c|}
\hline \multicolumn{2}{|c|}{$\begin{array}{l}\text { STIMULUS } \\
\text { AND \& CR }\end{array}$} & $\begin{array}{l}\text { CHARACTERS } \\
\text { CONFUSED }\end{array}$ & $\begin{array}{l}\text { STIMULUS } \\
\text { AND \& CR }\end{array}$ & \multirow{2}{*}{$\begin{array}{l}\text { CHARACTERS } \\
\text { CONFUSED } \\
6=3\end{array}$} & $\begin{array}{l}\text { STIMULUS } \\
\text { AND } \$ C R\end{array}$ & $\begin{array}{l}\text { CHARACTERS } \\
\text { CONFUSED } \\
\end{array}$ \\
\hline D & (89) & -- & G (77) & & M (70) & $N=8, K=3, K=3$ \\
\hline k & (89) & $\cdots$ & $6 \quad(77)$ & $8=4$ & J (69) & $K=4,0=3,8=3$ \\
\hline $\mathbf{R}$ & (89) & $\cdots$ & F (76) & $R=4$ & $N \quad(68)$ & $U=15$ \\
\hline 2 & (88) & $\cdots$ & H (76) & $\mathrm{N}=3, \mathrm{R}=3$ & C (66) & $G=8,2=3,0=3$ \\
\hline $\mathrm{T}$ & (86) & --- & $x \quad(76)$ & $K=4$ & s (65) & $5=20$ \\
\hline B & (85) & -- & $3(76)$ & $2=4$ & $2 \quad(65)$ & $2=18$ \\
\hline L & (84) & $\cdots$ & $9(76)$ & $8=7$ & $0 \quad(62)$ & $D=14, Q=10, D=3$ \\
\hline 5 & (83) & $S=4$ & 4 (74) & $2=4,8=3$ & $Q \quad(60)$ & $y=6,2=5,0=4, R=4, G=3$ \\
\hline $\mathrm{u}$ & (82) & $N=6$ & 7 (74) & $z=6,2=3$ & I (53) & $0=6,2=5,8=4$ \\
\hline $\mathbf{E}$ & (79) & --- & $P \quad(73)$ & $R=12, B=4$ & $v \quad(43)$ & $U=40, Y=3$ \\
\hline N & (79) & $M=5$ & A (72) & $R=11,2=3$ & 1 (35) & $I=15,2=10,8=6,0=5, K=4$ \\
\hline $\mathbf{Y}$ & (78) & $V=5, u=3$ & $8 \quad(72)$ & $B=4,2=3$ & (33) & $Q=14,2=10,8=9, \quad 0=6, \quad B=4, \quad X=3$ \\
\hline
\end{tabular}

Fig. 4. Percentage correct responses (CR) made to stimuli presented for recognition through an LED module. Incorrect responses (exceeding mean frequency of 2.8\%) to each test stimulus are listed as characters confused with the percentage responses to each. Test characters are listed in the order of least-to-most confused.

\section{REFERENCES}

advantageous to select LED modules in which the ROM is programmed with five additional 7-bit words representing $\mathrm{ON}$ functions for the 7 diodes in each column. The display of a more complete and discrete masking stimulus, such as a solid rectangular image, would then be possible. However, with high-speed sequential presentation of special characters (e.g., \#, H, and 1), a fairly complete masking stimulus is possible, even with those LED modules which do not otherwise provide for complete matrix illumination.

Haber, R. N. An on-line computer in a visual perception laboratory. Behavior Research Methods \& Instrumentation, $1968,1,86-93$

Massaro, $D$. W. Stimulus information vs processing time in auditory pattern recognition. Perception \& Psychophysics, $1972,12,50-56$.

\section{NOTE}

1. The MDA-III display module is manufactured by the Monsanto Company, Chicago, lllinois 60601 .

(Received for publication October 4, 1972; revision received November 13, 1972.) 